CAHIERS DE

NARRATOLOGIE
Cahiers de Narratologie

Analyse et théorie narratives

17 | 2009

Stéréotype et narration littéraire

\title{
Texte itératif et stéréotypes chez William Burroughs : de l'intertextualité à l'autostéréotypie
}

\section{Benoît Delaune}

\section{(2) OpenEdition}

Journals

Édition électronique

URL : http://journals.openedition.org/narratologie/1268

DOI : 10.4000/narratologie.1268

ISSN : 1765-307X

Éditeur

LIRCES

\section{Référence électronique}

Benoît Delaune, "Texte itératif et stéréotypes chez William Burroughs : de l'intertextualité à

l'autostéréotypie », Cahiers de Narratologie [En ligne], 17| 2009, mis en ligne le 18 décembre 2009,

consulté le 15 novembre 2019. URL : http://journals.openedition.org/narratologie/1268 ; DOI

10.4000 /narratologie. 1268

\section{Ce document a été généré automatiquement le 15 novembre 2019.}

\section{c) (†) $९$}

Cahiers de Narratologie - Analyse et théorie narratives est mis à disposition selon les termes de la licence Creative Commons Attribution - Pas d'Utilisation Commerciale - Pas de Modification 4.0 International. 


\title{
Texte itératif et stéréotypes chez William Burroughs : de l'intertextualité à l'autostéréotypie
}

\author{
Benoît Delaune
}

1 L'auteur américain William Burroughs (1914-1997) est connu pour son livre Le Festin nu (1959), puis pour sa technique du « cut-up » ou découpage de textes (qu'il a utilisée en gros de 1960 à 1968). Cet écrivain que Timothy S. Murphy place dans une zone grise entre modernité et post-modernité (voir son essai Wising Up The Marks sous-titré «The Amodern William Burroughs») a largement utilisé dans ses fictions, d'une part des emprunts littéraux ou indirects à la paralittérature (roman noir ou d'espionnage, science-fiction), d'autre part des procédés de ressassement d'un texte littéral ou tronqué, de livre en livre, selon ce qu'on pourrait qualifier d'autostéréotypie. Ayant lors de notre travail de thèse (2003) étudié certaines limites des théories de la transtextualité (Genette), de l'intertextualité (Kristeva) ou de l'autotextualité ou intratextualité (Ricardou, Jenny et Dällenbach), dès lors qu'on tentait de les appliquer à des textes résistants comme la "Trilogie» de Burroughs (The Soft Machine, The Ticket That Exploded, Nova Express, traduits sous les titres La Machine molle, Le Ticket qui explosa, Nova Express'), il nous paraît indispensable d'analyser ces textes sous l'angle de la stéréotypie (Jean-Louis Dufays) et de l'autostéréotypie (Stéphanie Orace). Ces deux modèles permettent de mieux envisager la prolifération du texte burroughsien, véritable "virus" mutant, et de tenter de théoriser les phénomènes de reprises littérales, tronquées, réécrites ou remontées, sur un corpus de textes déterminés. Dans le même mouvement, l'étude du texte cut-up burroughsien, par le fait qu'il est une sorte de texte-limite, peut permettre de compléter l'approche théorique de l'autostéréotypie.

2 Cette étude de l'autostéréotypie nous conduit également, logiquement, vers l'usage de figures paralittéraires dans la Trilogie et Le Festin nu de Burroughs. L'utilisation de figures stéréotypées chez Burroughs et les procédures de reprise et de réécriture sont indissociables chez cet auteur et font partie, semble-t-il, d'une véritable politique du 
texte. Ce type d'approche peut ainsi fournir des éléments d'explications extrêmement révélateurs, à la fois sur les phénomènes d'autostéréotypie et sur les techniques d'écriture d'un écrivain particulier que des auteurs aussi divers que Christian Prigent, Bernard Heidsieck, Claude Pélieu, Olivier Cadiot ou FJ Ossang, pour ne parler que du domaine français, considèrent comme une référence importante.

Les théories de la réécriture et de l'intertextualité chez Burroughs

Dès la publication de son premier livre d'importance, Le Festin nu (The Naked Lunch) en 1959 à Paris, William Burroughs a mis en place des techniques d'écritures bien précises qui ancrent le texte du côté de la paralittérature et de la figure de l'itération. Ce texte, sorte de canevas textuel constitué de fragments narratifs en apparence décousus, présente ainsi un curieux mélange de "carnets d'un désintoxiqué ", de pages proches du roman noir "hard-boiled ", de roman d'espionnage, ou même de certains thèmes lorgnant vers la science-fiction. Dès ce "roman " à l'inscription générique souvent contestée par William Burroughs lui-même se met en place une écriture gorgée d'éléments empruntés à la paralittérature, dans un bric-à-brac esthétique (et, pourrions-nous dire, quasiment éthique) qui a pour fonction, selon l'auteur, de libérer les mots et la langue de tout système répressif. Dans ce prolongement, William Burroughs a utilisé entre 1960 et, en gros, 1975, un système d'écriture radical, le procédé du « cutup ", découpage sauvage et accouplement de textes de toute origine, pages écrites de la main de Burroughs, pages du Coran ou de la Bible, pièces de Shakespeare, romans de Genet ou Kafka, poésies de Rimbaud, romans policiers ou de science-fiction... Le processus en place dans le cut-up pose alors évidemment la question de l'intertextualité, ainsi que celle du stéréotype, par d'une part le large emploi de thèmes et figures originaires des paralittératures, d'autre part par la répétition constante sur plusieurs livres de fragments textuels et de motifs narratifs selon un agencement réfléchi.

Ne le cachons pas, les textes burroughsiens de cette période, surtout ceux de la Trilogie des années 1960 (La Machine molle, 1960, Le Ticket qui explosa, 1961, Nova Express, 1964), posent d'énormes problèmes dès lors que l'on souhaite y appliquer les théories de l'intertextualité (aux sens genettien ou kristevien du terme) ou de l'intratextualité ou autotextualité (Ricardou, Jenny, Dällenbach) : pour dire la vérité, ces théories ne marchent qu'en partie sur des textes qu'on pourrait qualifier de résistants ${ }^{2}$. Ces textes résistent d'autant plus qu'ils ont connus des phases de réécriture selon des versions successives très différentes ${ }^{3}$.

5 Annick Bouillaguet, reprenant les catégories genettiennes, a schématisé en 1990 une «typologie de l'emprunt » selon le tableau suivant:

\begin{tabular}{|c|c|c|}
\hline & explicite & non-explicite \\
\hline littéral & citation & plagiat \\
\hline non-littéral & 介 référence & allusion $\Uparrow \mathbb{~}$ \\
\hline
\end{tabular}

6 Cette typologie, au demeurant très fonctionnelle sur la plupart des textes, pose déjà un problème de fond lorsqu'on considère le texte burroughsien : ainsi, lorsque dès Le Festin $n u$ un même fragment textuel de plusieurs lignes est réutilisé plusieurs pages plus loin, soit sous une forme littérale soit sous la forme d'une variante approchante, doit-on parler de « citation » et de "référence " ? Bien sûr, on nous répondra que nous sommes là face à une pratique de réécriture interne plutôt que d'intertextualité, la plupart des fragments semblant provenir de la main même de Burroughs. Les nombreuses variantes présentes dans les romans de Claude Simon par exemple peuvent fonctionner 
également selon le même ressort ; ainsi, la scène du cheval mort, répétée dans La Route des Flandres. Cependant, d'une part l'origine textuelle chez Burroughs pose un sérieux problème (est-on face à un texte de la main de l'auteur, ou à un emprunt?) ; d'autre part il faut noter que cette pratique de reprise d'un même type de texte, dès Le Festin $n u$, fait partie non pas d'une volonté explicite de l'auteur et d'une construction rigoureuse mais plutôt d'une politique surprenante de laisser faire par rapport au texte. Brion Gysin a raconté en 1975 la genèse éditoriale de ce livre, dont l'ordre des "sections" ou chapitres a été décidé par le hasard des renvois d'épreuve par l'imprimeur :

[...] déjà Naked Lunch n'était pas un roman comme les autres. Il n'y avait ni commencement, ni milieu, ni fin. Burroughs disait qu'on pouvait prendre son livre, l'ouvrir et le lire à partir de n'importe quel point d'interrogation. [...] Il y eut même un nombre effarant de versions du Festin nu, qui n'était pas un livre dans l'acception traditionnelle du terme, mais un véhicule en perpétuel mouvement ${ }^{4}$.

Il ajoute plus loin, à propos du processus d'édition de The Naked Lunch :

[...] nous devions non seulement corriger les coquilles, mais remettre tout en ordre. Il fallait bien en choisir un, mais lequel ? Puisqu'il n'y avait pas de classement à l'origine, nous avons dû procéder à une reconstitution totale du texte, mais en abandonnant la fichue linéarité conventionnelle du roman: Burroughs est ainsi parvenu à ce que nous pourrions appeler une sorte d'écrition ${ }^{5}$.

William Burroughs déclare lui-même par rapport au processus éditorial du livre :

La forme définitive de Naked Lunch et la juxtaposition de sections étaient déterminées par l'ordre dans lequel le matériel partait - au hasard - chez l'imprimeur ${ }^{6}$.

9 Cette politique d'indifférence face au matériau textuel et au support-livre en dit déjà long sur Burroughs : la réécriture ou l'intertextualité, chez lui, peuvent être le fruit d'un hasard ou d'une indécision plutôt qu'une démarche réfléchie. Des catégories comme "l'allusion" ou la "référence ", sont donc déjà problématiques face à cette vision du texte. Les catégories d'intertextualité ou de réécriture, elles aussi, peuvent poser problème.

10 La politique éditoriale quelque peu involontaire du Festin $n u$ trouve un prolongement surprenant dans le livre suivant de Burroughs, La Machine molle (The Soft Machine), où le texte est littéralement passé à la moulinette du procédé du "cut-up », technique de découpage du texte avec des ciseaux, suivant une ligne horizontale et une ligne verticale, donnant quatre sections réarrangées dans un ordre différent. Le procédé prend alors une ampleur inédite lorsque Burroughs découpe aussi bien ses propres textes que des pages de Rimbaud, du Coran, de journaux, de romans policiers, etc. Nous pouvons, de façon encore plus claire, poser ici les limites des théories intertextuelles ou de réécriture face à ce type d'écriture : en effet, le texte bascule très souvent dans le régime de l'emprunt littéral ou non-littéral, explicite dans certains cas, implicite dans d'autres, donc du côté de la citation, de l'allusion, du plagiat ou de la référence. Mais tous ces termes posent un réel problème. Ainsi, «citer » ou désigner par une allusion, c'est faire signe à un narrataire. Dans le cas de l'emprunt littéral implicite donc du plagiat, apparait par contre la volonté de cacher quelque chose, de ne pas faire signe. Mais dans tous les cas il est question de faire signe ou pas, de mettre en place une connivence ou non entre une instance auctoriale et un narrataire, et donc pour l'auteur de porter un jugement sur les éléments empruntés ou repris. Il apparaît que chez Burroughs, justement, le texte est soumis à un tel dépeçage, avec un tel morcellement 
des sources et un tel mépris affiché des origines, que parler de jugement porté sur les éléments empruntés est paradoxalement impossible. Le texte burroughsien semble de ce point de vue totalement désacralisé via la cisaille du cut-up. C'est bien ce que déclarait Burroughs à Allen Ginsberg dès 1959 dans Les Lettres du Yage, lorsqu'il écrivait qu' "aucun mot n'est sacré » et qu'il reprenait à son compte une devise attribuée à Hassan I Sabbah, maître de la secte islamiste des Haschichins : « Rien n'est vrai, tout est permis » (« Nothing is real, everything is permitted »).

William Burroughs, à quelques rares reprises, a publié des textes explicatifs sur le cutup, indiquant par exemple en légende les textes-sources et donnant dans le texte final l'origine de chaque $\operatorname{mot}^{7}$ :

(Ni ne pouvez échapper... Il n'y en a pas d'autres qu'Allah pour protéger [a])... (Alors que je traversais le désert c'était [jt]) : (Remarquant que vous avez ILS pensèrent débarrassés ceux qui rendent grâce [a])... (voir Ligne... Street Bone. Street Bone savait qu'il n'y avait pas moyen d'y échapper... Vous souvenez-vous du médium de mes doigts lointains? [wb])... (Dans les cieux pas une seule étoile sur terre nulle piste $[j t]) \ldots$

(Ne pas corrompre la Volonté d'Allah en redoutant vos actions antérieures [a])... (cela mon âme est en moi composée de traîtrise... Un jeune Vaisseau Chante condamné à mort par les Forces Navales [jg])...(Rien que ça aurait dû être évident photo à l'aube singe faffeur... Os morts [wb])... (Air caillé [jt])... (Interelation avec une vie dans laquelle je me suis retrouvé mêlé [jg])... (Ne pas corrompre la Volonté d'Allah en redoutant vos actions antérieures [a])... (Passé à toute allure ailes brinquebalantes... Elle mangeait $[j t])[. .$.

Légende :

a : Allah

$\mathrm{b}:$ Bible

wb : William Burroughs

jg: Jean Genet

jt: James Thompson

ws : William Shakespeare

hs : Hassan I Sabbah"

Hormis l'ironie de cette « légende » qui nous montre que le texte de Burroughs côtoie sans complexe la Bible ou Genet, le procédé de découpage atteint ici une ampleur inédite car c'est l'un des rares textes de Burroughs où la provenance textuelle est aussi visible (non seulement par la légende, mais aussi par les fragments utilisés, fortement typés, à l'image de ce « Ne pas corrompre la Volonté d'Allah en redoutant vos actions antérieures »). Car dans la plupart des textes cut-up postérieurs, les textes sources deviennent insituables, à l'image ici de l'expression «elle mangeait» empruntée au poète anglais James Thompson! Ce genre d'emprunt serait sans doute extrêmement dur à déterminer si Burroughs ne nous indiquait pas la source... C'est bien, dans l'absolu, un emprunt «littéral», donc un plagiat, mais qui s'opère à un niveau tellement microscopique qu'on ne peut plus vraiment parler ni de plagiat, ni d'allusion, de citation ou de référence. Ce cas limite montre bien que Burroughs, dans sa politique textuelle, se soucie peu de faire signe ou pas. Ce qui importe pour lui, c'est, bien plus qu'un subtil jeu de références faisant écho à une critique des sources, le résultat, l'après-découpage. Bien sûr, découper Shakespeare, un poète anglais du XIX ou la Bible et les accoupler à Genet, c'est porter un jugement sur les textes de départ, faire acte de vandalisme par rapport à l'écrit, aux notions de littérature, de culture. Mais au-delà de cet acte d'accouplement, le texte final ne prétend pas faire signe ; au contraire il s'en moque totalement. Dès lors que le "jugement" a été porté sur les textes dans la 
procédure de départ, dans l'intention, la notion de faire référence, faire signe, tombe: Burroughs ne prétend pas mettre en scène une culture encyclopédique ou railler tel ou tel texte précisément, il se pose plutôt, avec l'utilisation de cette technique à fonction "indifférenciante» qu'est le cut-up, comme un fossoyeur de l'écrit et de la "Littérature ». La transtextualité ou l'intertextualité, c'est-à-dire la mise en rapport de textes les uns avec les autres, ne l'intéresse pas vraiment si ce n'est pour inoculer une sorte de "virus » à l'écrit : «le mot est un virus ", déclare-t-il d'ailleurs à plusieurs reprises $^{8}$.

Le début du livre d'entretien Le Job est à cet égard significatif de l'attitude de Burroughs par rapport à la littérature : à George Steiner le prenant à parti dans un article pour ses «descriptions franches et explicites de scènes sexuelles » et s'exclamant: « Au nom de l'intimité humaine, assez!», Burroughs répond: «Au nom de qui évoque-t-on là l'intimité humaine? Au nom de ceux qui cachèrent des micros dans la chambre à coucher de Martin Luther King ${ }^{9}$ [...] ? » Ici deux mondes s'affrontent, d'un côté George Steiner, écrivain et fin lettré, parle de l'écriture, de la littérature, de ce qu'elle doit dire et de ce qu'elle doit cacher en tant que représentation du monde. De son côté Burroughs répond sur un plan totalement différent, ouvertement politique, en évoquant l'affaire du Watergate, mettant sur le même plan des faits de la vie civile et politique et son écriture. Cet exemple vient éclairer la conception presque a-littéraire qu'a Burroughs de l'écriture; la notion d'intertextualité, avec ses jugements "d'allusion » ou de "référence », ne s'applique finalement qu'à des auteurs empreints d'une certaine vision de la littérature et de la culture, une vision «littéraire " que Burroughs n'a pas ou ne veut pas avoir. Pour lui, l'écriture est un combat contre le "virus » qu'est le mot écrit, elle doit « rendre compte » et dénoncer les dérives des systèmes politiques, l'écrivain écrit d'ailleurs, avant même la découverte du cut-up, dès la fin des années 50 :

Insatisfaction complète de tout ce que j'ai fait comme écriture... si l'écriture n'a pas le danger et l'immédiateté, l'urgence de la tauromachie, elle n'a rien à voir avec la façon dont je la conçois... je suis fatigué d'être placé derrière les lignes avec un appareil d'enregistrement imparfait qui reçoit des bulletins inexacts... je dois atteindre le front ${ }^{10}$

C'est pourquoi, face à une telle conception du fait textuel, il faut sans doute rechercher du côté d'autres approches théoriques, afin de mieux rendre compte des processus en marche dans l'écriture de William Burroughs. Ainsi, au-delà de la seule notion d'intertextualité, il convient d'observer en quoi le texte burroughsien opère du côté de la répétition sans fin d'un matériau textuel réutilisé selon de multiples arrangements, sous-tendue par une conception implicite de motifs stéréotypiques ou autostéréotypiques.

Une écriture du ressassement et du stéréotype

Au-delà de l'emploi du procédé du cut-up, William Burroughs a développé dans ses livres tout un arsenal de techniques basées sur la répétition d'un même matériau, à l'intérieur d'un corpus de textes fonctionnant ensemble (par exemple les trois livres de la Trilogie avec, dans une moindre mesure, ce que nous qualifions de "quatrième tome " fantôme de la Trilogie, le livre Dead Fingers Talk publié en 1963 en Grande Bretagne uniquement) comme dans des ensembles plus hétérogènes (comme le montre la dizaine de textes composés par Burroughs entre 1965 et 1975 autour du discours délirant du gangster Dutch Schultz lors de son agonie). Observer la mise en place ce texte répétitif, par le biais de topoï spécifiquement burroughsiens, peut permettre ensuite de 
déterminer plus exactement la vision qu'a Burroughs du texte. C'est pourquoi il est intéressant de se pencher sur les stratégies de Burroughs par rapport à une certaine forme d'intratextualité ou d'autotextualité, et d'observer de quelle manière celui-ci construit tout un discours sur la réutilisation de textes et de schémas narratifs antérieurs, par la reprise de stéréotypes et la mise en place d'une " autostéréotypie ». La Trilogie fonctionne selon la multiplication de fragments textuels récurrents, reprises littérales ou découpées puis remontées d'un matériau souvent antérieur. Cette répétition du texte, au niveau des mots, s'accompagne également d'une répétition de motifs (empruntés le plus souvent à la paralittérature). À la suite de Jean-Louis Dufays et Stéphanie Orace, nous nommons ce procédé itératif global l'autostéréotypie. JeanLouis Dufays, dans son ouvrage Stéréotype et lecture ${ }^{11}$, avance un constat très simple et très éclairant : le stéréotype, bien que très mal considéré par la plupart des auteurs comme par la plupart des critiques, est pourtant le processus présent à la base de toute production de signification. La langue même est un ensemble de stéréotypes, comme l'écrit Dufays, elle est " par définition le système de stéréotypes le plus stable qui soit ${ }^{12}$ ». Loin d'être le signe d'une absence de valeur littéraire, le stéréotype est donc un élément indispensable à la construction (et l'acquisition pour le lecteur) d'un texte. Il est l'unité macrotextuelle qui détermine la lecture, selon l'interaction entre le texte et ses contextes. La stéréotypie permet donc au lecteur d'avoir en quelque sorte des points de repère - par rapport à sa propre culture et son propre contexte, bien évidemment - dans un texte.

17 La récurrence textuelle s'inscrit donc en général dans un mouvement naturel du texte. Avec Burroughs, nous voyons tout de même que celle-ci est nettement développée, et dépasse les seuls critères sémiologiques de la production de signification. De plus, comme Burroughs ressasse dans la plupart de ses livres un matériau toujours voisin, voire identique, il faut bien parler d'une utilisation en quelque sorte en circuit fermé de la stéréotypie, comme le montrent ces trois passages issus de trois livres différents :

The Yage Letters (lettre datée de 1953) :

Bill Gains was in town and he has burned down the Republic of Panama from Las Palmas to David on Paregoric ${ }^{13}$.

Bill Gains était en ville et il a cramé la république de Panama de Las Palmas à David avec le Parégorique ${ }^{14}$. [nous traduisons]

The Naked Lunch (1959) :

[Bill] Gains and Lee burned down the Republic of Panama from David of Darien on paregoric ${ }^{15}[. .$.

[Bill] Gains et Lee ont grillé toute la République de Panama, de David à Darien, en carburant au parégorique ${ }^{16}[\ldots]$

The Soft Machine (1961):

The Sailor And I burned down The Republic of Panama from Darien swamps to David trout streams on paregoric and goof balls-(Note :Nembutal) ${ }^{17}$

Le matelot et moi avons brûlé la République de Panama, des marécages de Darien aux torrents à truites de David, en carburant au Parégorique et aux somnifères (Note : Nembutal ${ }^{18}$ )

Cet exemple nous montre bien que le peu de variations du matériau utilisé, si l'on considère que les mêmes groupes de mots peuvent revenir plus d'une centaine de fois dans la Trilogie, entrâne une certaine forme de monotonie (le mot n'est pas ici péjoratif) qui confine à la ritualisation, à l'égrenage d'un texte dont les éléments peuvent permuter de temps à autre mais restent à peu près les mêmes. 
genettiennes de la citation, du plagiat et surtout de l'allusion. Ayant déjà, dans notre propre travail de thèse, émis certaines réserves vis-à-vis de ces catégories qui ne s'appliquent pas aussi facilement à un texte-limite comme la Trilogie, nous pouvons dire que la reprise d'un texte chez Burroughs, qu'elle soit littérale ou non, met en marche une forme visible et ostensible d'intratextualité, puisque le lecteur, suivant sa «culture » du texte, peut reconnaître tel ou tel fragment qu'il a déjà lu, à plusieurs degrés, du plus visible au quasi invisible. La répétition, sous une forme littérale ou fragmentée, permet également la mise en place de tout un travail d'autostéréotypie au sens large, puisque le texte se structure suivant le procédé itératif qui rajoute du sens. Là où le texte burroughsien apporte quelque chose de différent par rapport, par exemple, à Claude Simon (dont les romans sont l'objet d'étude de Stéphanie Orace), c'est qu'il est certain que quelqu'un n'ayant lu qu'un des trois livres de la Trilogie sera capable tout de même de ressentir et de recevoir ces répétitions constantes dans le texte; alors que pour Stéphanie Orace « un lecteur vierge d'autres romans simoniens ne percevra pas ce phénomèn ${ }^{23} »$ [de reprise d'un motif].

L'écriture de Burroughs diffère donc car, pourrait-on dire, l'auteur impose au lecteur ces répétitions du texte, là où Claude Simon pour Stéphanie Orace travaille du côté de la « connivence ${ }^{24}$ » entre l'auteur et un lecteur «familier de [son] univers ${ }^{25}$ » (nous sommes là du côté de l'allusion genettienne). Lorsque chez Burroughs sont répétées constamment, selon un ordonnancement différent et une fréquence variable, certaines expressions, par contre nous sommes dans l'explicite. On peut avancer que la Trilogie est constituée sans doute en grande partie à partir d'un Ur-texte hypothétique, un corpus textuel indéfini qui aurait servi de réservoir de mots ou de fragments textuels, combinés, recombinés selon des processus exponentiels de découpage/remontage/ redécoupage/re-remontage.

Voici ci-dessous un exemple parmi les plus significatifs, tiré de Nova Express (le troisième tome de la Trilogie), où deux passages de The Soft Machine, eux-mêmes déjà réutilisés dans ce même livre à plusieurs reprises sous une forme littérale ou tronquée, forment le matériau de base de deux pages constituées à plus de la moitié de mots repris de ces deux textes sources : 


\begin{abstract}
So many years - that image - got up and fixed in the sick dawn-No me hagas caso-Again he touched like that - smell of dust-The tears gathered-In Mexico again he touched-codeine pills powdered out into the cold Spring air - Cigarette holes in the vast Thing Police Could give no information other than wind idenfity fading out - dwindling "Mr. Martin" couldn't reach is all-Bread knife in the heartShadow turned off the lights and water-We intersect on empty walls-Look anywhere-No good-Falling in the dark mutinous door-Dead Hand stretching zero-Five times of dust we made it all the living and the dead-Young form went to MadridDemerol by candlelight-Wind hand-The Last Electrician to tap on pane-Migrants arrival-Poison of dead sun went away and sent papers-Ferry boat cross flutes of Ramadan-Dead muttering in the dog's space-Cigarette hole in the dark-give no information other than the cold Spring cemetery-The Sailor went wrong in corridors of that hospital-Thing Police keep all Board Room Reports is all-Bread knife in the heart proffers the disaster accounts-He just sit down on "Mr. Martin"-Couldn't reach flesh on Niño Perdido-A long time between flutes of Ramadan-No me hagas caso sliding between light and shadow-

"The American trailing cross the wounded galaxies con su medicina, William."

Half your brain slowly fading-Turned off the lights and water-Couldn't reach flesh empty walls-Look anywhereDead on tracks see Mr. Bradly Mr. Zero-And being blind may not refuse the maps to my blood whom I created"Mr. Bradly Mr. Martin," couldn't you write us any better than that?-gone away-You can look any place-No good-No bueno-
\end{abstract}

\title{
Légende des caractères utilisés : Corpus A', mots provenant de A (The Soft Machine, pp. 8-11) Corpus B', mots provenant de B (The Soft Machine, p. 178)
}

$\mathrm{X}$, mots de provenance indéterminée

Nova Express, pp. 28-29; origines du matériau textuel d'un texte cut-up.

Tant d'années-cette image-levé et shooté dans l'aube malade-No me hagas casoÀ nouveau il a touché comme ça-odeur de poussière-Les larmes s'agglutinèrentÀ Mexico à nouveau il a touché-pilules de codéine saupoudrées dans l'air froid du printemps-Trous de cigarette dans la vaste Police des Choses-Ne put donner d'autre information que l'identité de vent s'évanouissant-s'amenuisant-«Mr. Martin » ne pouvait atteindre est tout-Couteau à pain dans le cœur-Ombre coupa les lumières et l'eau-Nous croisons sur des murs vides-Regarde n'importe où-Pas bon-Tombant dans la porte rebelle sombre-Main Morte allongeant zéro-Cinq fois de poussière nous avons baisé tous les vivants et les morts-Forme Jeune alla à Madrid-Demerol à la lueur d'une bougie-Main du vent-Le Dernier Électricien à taper sur la vitre-Arrivée de migrants-Poison de soleil mort partit et envoya des papiers-Ferry croise flûtes de Ramadan-Mort maugréant dans l'espace du chienTrou de cigarette dans la nuit-ne donne pas d'autre information que le cimetière du printemps froid-Le Matelot a mal fini dans les corridors de cet hôpital-La Police des Choses garde tous les Rapports de la Chambre des Livres est toutCouteau à pain dans le cœur profère les comptes du désastre-Il s'assied juste sur «Mr. Martin »-Ne pouvait atteindre la chair sur Niño Perdido-Un long moment entre les flûtes du Ramadan-No me hagas caso glissant entre ombre et lumière"L'Américain traînant à travers les galaxies blessées con su medicina, William. » La moitié de ton cerveau s'évanouissant lentement-a coupé les lumières et l'eauNe pouvait atteindre la chair-murs vides-regarde n'importe où-Mort sur les rails vois Mr. Bradly Mr. Zéro-Et étant aveugle ne pouvais refuser les planisphères à mon sang que j'ai créé-«Mr. Bradly Mr. Martin», ne pouvais-tu pas m'écrire 
mieux que ça ?-"Parti-Tu peux regarder n'importe quel endroit-Pas bon-No bueno- [Nous traduisons] ordre différent. La formulation, sous forme d'un texte-slogan injonctif, voire d'un texte télégraphique (avec l'emploi répété de participes présent), vient renforcer cette idée de réservoir de mots. D'ailleurs, des expressions comme "Shift linguals " proviennent en droite ligne du premier livre cut-up de 1960, Minutes To Go, recueil collectif à valeur de manifeste, co-signé par Burroughs, Brion Gysin, Gregory Corso et Sinclair Beiles. La réutilisation constante tout au long des années 1960 d'expressions qui apparurent avec les premiers essais de cut-up nous conforte dans l'idée que Burroughs utilisait sciemment des textes sources bien définis, un ensemble constituant comme une forme de Ur-texte.

31 Autre exemple encore plus significatif, les thématiques de l'injection de drogue ou de la maladie liée au manque entraînent le retour également d'un même type de séquence, dont les phrases sont découpées et remontées avec un matériau différent. Par exemple, dans Junky, un personnage, le vieil Ike, parle au narrateur en ces termes :

«I'm going to quit, » I said aloud.

I made up a solution of hop and told Ike to stay away for a few days. He said, «I hope you make it, kid. I hope you get off. May I fall down and be paralized if I don't mean $\mathrm{it}^{26} . »$ 
- Je vais laisser tomber, dis-je à haute voix.

Je préparai une solution d'opium et demandai à Ike de ne plus se montrer pendant quelques jours. Il me dit :

- J'espère que tu y arriveras, petit. J'espère que tu vas arrêter. Je veux bien être pendu si je ne suis pas sincère ${ }^{27}$.

Le même passage se retrouve mot à mot dans The Soft Machine :

«You're quiting? Well I hope you make it, kid. May I fall down and be paralized if I don't mean it... You gotta friend in me. A real friend and if ${ }^{28}$ ”

"Tu décroches? Eh bien j'espère que tu y arriveras, fiston. Que je tombe paralysé si je ne suis pas sincère. T'as trouvé un ami. Un vrai ami et si » [nous traduisons]

On le voit, la réplique de Ike dans Junky est reprise telle quelle, à l'exception d'une phrase retranchée («I hope you get off. »), dans la réplique du narrateur de la première section de The Soft Machine. Ceci confirme le fait que Burroughs, dans la Trilogie, utilise certes un matériau en partie emprunté à d'autres auteurs, mais surtout qu'il puise en grande partie dans son propre matériau textuel, comme s'il y avait volonté d'inventer ses propres stéréotypes. Un autre exemple d'intersection intéressante peut être relevé entre la postface de The Naked Lunch et la première section de The Soft Machine:

Pushing in a small way to keep our habit...

" And use that alcohol, » I said slamming a spirit lamp down on the table.

"You fucking can't - wait - hungry junkies all the time black up my spoons with matches... That's all I need for pen Indef. the heat rumbles a black spoon in the $\operatorname{trap}^{29} \ldots$

Fourguer la came à la petite semaine pour entretenir son propre besoin...

- et flambe-la à l'alcool, dis-je en flanquant la lampe à mèche sur la table. Vous êtes donc pas foutus d'attendre?... sales voraces... tous des emmerdeurs qui me noircissent le cul de mes cuillers avec des allumettes... tout ce qu'il me faut pour aller au trou c'est que la poulaille tombe sur une cuillère noircie dans ma piaule ${ }^{30} .$.

Ce fragment, présent à la toute fin de The Naked Lunch, se retrouve quasiment mot à mot dans The Soft Machine, créant une sorte de lien entre les deux livres :

Pushing in a small way to keep The Habit: INVADE, DAMAGE, OcCUPY. Young faces in blue alcohol flame.

" And use that alcohol. You fucking can't wait hungry junkies all the time black up my spoons. That's all I need for Pen Indef. the fuzz rumbles a black spoon in my trap.» The old junky spiel ${ }^{31}$.

Fourguant un minimum pour entretenir l'Habitude: ENVAHI, ENDOMMAGE, OCCUPE. Jeunes visages dans une flamme bleue d'alcool.

«Et sers-toi de cet alcool. Vous ne pouvez pas attendre putains de camés affamés, tout le temps à noircir le cul de mes cuillères. C'est tout ce qu'il me faut pour que Pen Indef le poulet renifle une cuillère noircie dans ma piaule. » Le vieux baratin des camés. [nous traduisons] symbolique : il souligne bien le fait que nous sommes dans un univers codé, l'univers de la came, qui fonctionne par itération. À cet univers de répétition («le sablier de la came ", comme le nomme Burroughs) se doit de correspondre une écriture itérative, toute faite de reprises à peine variées de phrases, de «baratin» («spiel»). Ce « vieux baratin des camés » est peut-être aussi l'occasion d'un clin d'œil au premier livre de Burroughs, justement nommé Junky. « The old junky spiel » prend alors une résonance particulière.

Il ne fait aucun doute, au vu des fragments que nous venons d'étudier, que chaque livre est constitué d'un matériau puisé en grande partie aux sources même des tout premiers 
textes de Burroughs. Ce matériau part bien des premières traces d'écriture de The Naked Lunch, qui remontent elles-mêmes à la période de Junky, Il est évident que ce matériau, ce réservoir textuel, est à la base de la Trilogie, et surtout de The Soft Machine.

De nombreuses similitudes, de l'ordre de la phrase, sont donc bien visibles entre la Trilogie et les livres antérieurs de Burroughs. Le procédé permet à Burroughs de mettre en œuvre une certaine forme d'unité textuelle, basée non pas sur la fragmentation d'un seul et unique récit, mais plutôt sur l'éparpillement de "routines» et sections qui entrent en résonance les unes les autres par l'intermédiaire de phrases récurrentes et donc d'autostéréotypes. Pour citer un dernier exemple, on peut noter une phrase qui revient à plusieurs reprises dans The Naked Lunch, aux pages 157 et 160 et qu'on retrouve mot à mot dans The Soft Machine:

The subway sweeps by with a blast of iron ${ }^{32}$.

Traduit par Mary Beach et Claude Pélieu par :

Le métro fonce dans une explosion de fer noir ${ }^{33}$

Par cette apparition de motif, on peut dire que Burroughs rythme son texte consciemment. Est également intéressant le fait que dès The Naked Lunch apparaît ce procédé de récurrence textuelle, comme si le passage au cut-up n'était alors qu'une étape logique dans l'arsenal scripturaire burroughsien. L'écriture de Burroughs, dès The Naked Lunch, est bien basée sur l'itération. Il s'agit pour l'auteur de créer ses propres stéréotypes, qui par leur fréquence parsèment le texte de jalons. Nous sommes du côté d'une technique de montage qui permet, à l'instar d'un film, la reprise, selon des cadrages identiques ou différents, d'une scène, d'un décor, suivant le principe d'une économie de moyens. Pour reprendre une image cinématographique, Burroughs opère dans la Trilogie et dès The Naked Lunch par juxtaposition de fragments narratifs, de façon lâche et imprévisible, à la manière d'un montage elliptique.

Ces exemples montrent également que bien au delà de la seule reprise du texte, se trouve la volonté de produire un message à la limite du bruit blanc, de l'entropie. Le texte peut devenir tout à coup chaotique et difficilement intelligible. Burroughs semble axer l'emploi du cut-up sur une vision précise et générale de l'écriture, qui se doit d'englober toutes les écritures qui la précèdent et se reproduire littéralement, ainsi qu'elle reproduit des textes d'autres auteurs, dans un mouvement de chaos, de multiplication et de prolifération.

Conclusion

41 Pour être complets, il nous faudrait également étudier l'utilisation par William Burroughs, tout au long de la Trilogie, d'images, de thèmes et de procédés narratifs provenant en droite ligne de la paralittérature. Car l'instabilité revendiquée du texte s'exprime aussi par la récupération et le détournement de schémas narratifs ou littéraires, de stéréotypes ou de clichés empruntés à la paralittérature, qui, en fournissant en apparence un semblant de stabilité, sont pourtant également employés afin de montrer une certaine dégénérescence du texte. La Trilogie fonctionne bien par la récupération de stéréotypes, ce mouvement d'assimilation où le cut-up devient l'instrument d'une appropriation de toutes les littératures, qu'il phagocyte pour en restituer une version distordue et apocalyptique. La volonté revendiquée chez William Burroughs de faire du neuf, de tourner le dos à cette forme " dépassée " que serait le romanesque, passe donc par l'utilisation du cut-up, mais également par l'intégration d'éléments empruntés à toutes les formes de littérature, dont des formes paralittéraires comme le roman policier ou la science-fiction. 

détournement de ses marges, de la paralittérature. La science-fiction ou le roman policier, constituant des genres extrêmement codifiés, sont utiles dans l'entreprise de désacralisation de la littérature qui anime Burroughs. Le texte stéréotypique emprunté à la paralittérature, qui va contre une littérature que l'auteur considère comme officielle, faisant partie de l'attirail d'instruments de contrôle de ceux qui ont le pouvoir, permet d'abaisser celle-ci, de la pervertir pour lui faire dire autre chose que son discours convenu. D'où l'accumulation d'éléments empruntés à la science-fiction, en commençant par Nova Express où il est question d'une planète en train d'exploser, et où les personnages voyagent en vaisseau spatial de galaxie en galaxie. Le fantastique apparait également avec les nombreuses transformations corporelles dont il est constamment question dans la Trilogie.

L'emploi de telles figures, dans un cynisme grinçant, permet à Burroughs la mise en place de tout un carnaval de personnages, une vraie galerie de music-hall - le terme de routine employé par Burroughs, d'ailleurs, y renvoie, nous l'avons vu. Il s'agit bien là d'intégrer à une littérature considérée comme expérimentale des éléments populaires, afin de subvertir l'image de la littérature académique. Apparaît peut-être également la volonté, à partir de la Trilogie, d'intégrer toutes sortes de textes, en quelque sorte tous les textes, dans un élan presque alchimiste. Burroughs, à travers le cut-up et la reprise aussi bien de textes de Genet que d'auteurs inconnus de science-fiction, cherche peutêtre à créer un sorte de Livre mallarméen d'une nouvelle espèce. C'est en tout cas ce qu'il déclare plus ou moins, dans un entretien avec Philippe Mikriammos de 1974 : «Je pense que je travaille encore dans le sens d'un mythe pour l'ère de l'espace et que tous mes livres constituent pour l'essentiel un seul livre ${ }^{34} »$.

À travers le cut-up, l'autostéréotypie et l'emploi de la paralittérature, il y a sans doute une vision singulière du texte. Le concept d'une "mythologie pour l'ère de l'espace ", employé à de multiples reprises par Burroughs, lui sert à justifier sa volonté de faire entrer la littérature dans le futur (ou le présent, car il considère que nous n'y sommes pas), et l'utilisation de la paralittérature comme du cut-up lui permet de se sentir, en tant qu'écrivain, en quelque sorte en phase avec son temps. Les techniques scripturales qu'il emploie sont donc le moyen d'entamer une rupture avec ce qui s'est fait précédemment, et d'emmener l'écriture sur des territoires inconnus. Derrière cette utilisation du stéréotype et d'une littérature en marge, se dessine même plus largement la remise en cause du savoir officiel, voire de tous les savoirs. Mais il faudrait un autre article pour étudier plus en profondeur ce rapport au savoir, à la culture et à la littérature, qui passe par un usage bien précis de la paralittérature et du stéréotype.

\section{BIBLIOGRAPHIE}

I] Textes de William Burroughs étudiés (versions originales et traductions, dans l'ordre chronologique de publication): 
Junky, Penguin Books, New York : 1977, préface d'Allen Ginsberg. Cette édition est la première qui soit complète, et sous le nom de William Burroughs.

Le Camé, Belfond, 1979 (traduction revue par P. Mikriammos).

Naked Lunch, Olympia Press (coll. « The Traveler's Companion »), Paris : 1959 ; Grove Press, NewYork : 1962 ; Calder \& Boyars, Londres : 1964. Repris en Grande Bretagne chez Paladin en 1986 (l'édition que nous utilisons).

Le Festin nu, Gallimard (coll. « du monde entier »), Paris, 1964. Traduction d'Eric Kahane.

The Soft Machine, Olympia Press (coll. « The Traveller's Companion »), Paris : 1961. Une deuxième version remaniée est publiée par Grove Press, New York : 1966 (l'édition que nous utilisons). Une troisième version, là encore remaniée, paraît en 1968 à Londres chez Calder \& Boyars.

La Machine molle, traduction par Claude et Mary Beach-Pélieu de la deuxième version, Christian Bourgois, Paris : 1968.

The Ticket That Exploded, Olympia Press, Paris : 1962. Une version légèrement remaniée paraît en 1963, toujours chez Olympia. Une troisième version, plus largement remaniée, paraît en 1967 chez Grove Press (l'édition que nous utilisons). La quatrième et dernière version est publiée à Londres en 1968 par Calder \& Boyars.

Le Ticket qui explosa, traduction de Claude et Mary Beach-Pélieu de la troisième version, Christian Bourgois, Paris : 1969.

The Yage Letters, par William Burroughs et Allen Ginsberg, City Lights Books, San Francisco : 1963.

Les Lettres du Yagé, publiées dans le Cahier de l'Herne Burroughs-Pélieu-Kaufman (coll. « L'écriture des vivants »), Paris : 1967. Traduction de Claude et Mary Beach-Pélieu (l'édition que nous utilisons). Reprises en volume aux éditions de l'Herne (coll. « Les livres noirs »), en 1970.

Dead Fingers Talk, John Calder Publisher, Londres : 1963. Ce livre n'a jamais été traduit en France.

Nova Express, Grove Press, New York : 1964 (l'édition que nous utilisons) ; Panther Books, Londres, 1969.

Nova Express, traduction de Claude et Mary Beach-Pélieu ; paraît aux pages 29-106 du Cahier de l'Herne Burroughs/Pélieu/Kaufman (coll. «L'écriture des vivants), éditions de l'Herne, Paris : 1967. Réédité en volume simple par Christian Bourgois, Paris : 1970.

Entretiens avec Daniel Odier, suivis de textes, Belfond, (coll. « Entretiens »), Paris, 1969 - réédition en 1979, sous le titre Le Job, augmentée et revue par Philippe Mikriammos, préface de GérardGeorges Lemaire.

The Job, Grove Press, New York : 1970.

Euvre croisée, Flammarion, (coll. «Connections »), Paris : 1976 (en collaboration avec Brion Gysin). Traductions de Gérard-Georges Lemaire, Christine Taylor, Jean Chopin. Préface de G.G. Lemaire.

Trilogie (La Machine molle, Le Ticket qui explosa, Nova Express), traductions par Claude et Mary Beach-Pélieu, Christian Bourgois, Paris : 1994.

II] Sur William Burroughs :

BOYER, Alain-Michel, Les Jeux de la réécriture : la fonction de la paralittérature dans les œuvres de William S. Burroughs, Alain Robbe-Grillet et Manuel Puig, doctorat de troisième cycle de littérature comparée, sous la direction de Pierre Brunel, 1981, Université de Paris IV-Sorbonne, pp. 20-21. 
GYSIN, Brion, Rub Out The Words, entretien avec G.G. LEMAIRE, in Revue d'Esthétique, 1975, n 3-4, « Il y a des poètes partout » pp. 184-204.

MIKRIAMmos, Philippe, William S. Burroughs, la vie et l'œuvre, Seghers (coll. « Littérature »), Paris : 1975.

MURPHY, Timothy S., Wising Up The Marks, The Amodern William Burroughs, University of California Press, Berkeley-Los Angeles-London : 1997.

osSANG, FJ, William Burroughs vs formule-mort, Jean-Michel Place (coll. « poésie »), Paris : 2007.

PARKINSON, Thomas (edited by), A Casebook On The Beat, Crowell, New York : 1961.

III] Textes théoriques :

BOUILHAGET, Annick, Une typologie de l'emprunt, Poétique n 80, 1990, pp. 489-497.

DÄLLENBACH, Lucien, Intertexte et autotexte, Poétique n²6, 1976, pp. 282-296.

DUFAYS, Jean-Louis, Stéréotype et lecture, Mardaga (coll. « Philosophie et langage »), Liège : 1994.

GENETTE, Gérard, Palimpsestes, Seuil : Paris, 1982.

ID, Nouveau discours du récit, Seuil (coll. « Poétique »), Paris : 1983.

ISER, Wolfgang, L'Acte de lecture, théorie de l'effet esthétique, Mardaga, Liège : 1985.

JAUSS, Hans-Robert, Pour une esthétique de la réception, Gallimard (coll. « Tel »), Paris : 1978.

JENNY, Laurent, La Stratégie de la forme, Poétique n²6, 1976, pp. 257-281.

KRISTEVA, Julia, Semeiotike, Seuil, (coll. « Tel Quel »), Paris : 1969.

ID, Le Texte du roman, Mouton-De Gruyter, La Haye-Paris : 1970.

MAINGUENEAU, Dominique, Le Contexte de l'œuvre littéraire, Dunod, Paris : 1993.

ORACE, Stéphanie, Éléments pour une autostéréotypie, Poétique n 125, Seuil, Paris : février 2001, pp. $17-31$.

RICARDoU, Jean, Pour une théorie du nouveau roman, Seuil, Paris : 1971.

ID, 'Claude Simon', textuellement article publié dans les actes du Colloque de Cerisy Claude Simon, 10/18, Paris : 1975, pp. 7-38.

\section{NOTES}

1. Nous utilisons, pour les versions en texte original, celles des éditions Grove Press. Pour la traduction nous utilisons par commodité (lorsque nous ne traduisons pas nousmêmes) la version des éditions Bourgois, qui réunit les trois livres dans un seul volume portant le titre de Trilogie.

2. Notre travail de thèse (2003) le démontre dans sa première partie : pour analyser la Trilogie burroughsienne à la lumière des théories de la transtextualité ou intertextualité, il faut revenir à la source par exemple de cette notion d'intertextualité (Kristeva, 1968). Et la multiplication des figures intertextuelles et des emplois de l'intertextualité chez Burroughs conduit en apparence à une impasse.

3. Par exemple The Soft Machine a connu deux réécritures, l'une de 1966, l'autre de 1968. La version la plus répandue actuellement, contrairement à ce qu'on pourrait penser, est la version de... 1966, transitoire ! 
4. « Rub Out The Words », pp. 188-189.

5. Ibid., p. 189.

6. Traduit par Jean Chopin dans l'Euvre croisée, dans le texte «Cut-Ups : projet pour une réussite catastrophique » de Brion Gysin, p. 54.

7. Texte publié dans G-G. Lemaire, Burroughs, Henri Veyrier (coll. « les plumes du temps »), Paris, 1986, pp. 63-65. Traduction de G-G. Lemaire.

8. Entre autres dans Le Job, Belfond, 1979, « Playback d'Eden à Watergate », p. 26.

9. Op. Cit., p. 26.

10. Lettre de Burroughs à Alan Ansen, citée par Alan Ansen, Anyone Who Can Pick Up a Frying Pan Owns Death, édité dans Big Table \# 2, Vol. 1 (Summer, 59), pp.32-41; repris dans A Casebook on the Beat, New York, 1961, pp.107 à 113.

11. Jean-Louis Dufays, Stéréotype et lecture, Mardaga (coll. « Philosophie et langage »), Liège : 1994.

12. Jean-Louis Dufays, op. cit., p. 31.

13. The Yage Letters, p. 3.

14. Les Lettres du Yage, in Cahier de l'Herne Burroughs - Pélieu-Kaufman. Traduction originelle de Claude Pélieu et Mary Beach, p. 3, nous traduisons pour garder un sens plus littéral.

15. The Naked Lunch, p. 173.

16. Le Festin nu, p. 239.

17. The Soft Machine, p. 33.

18. Trilogie, p. 48.

19. Stéphanie Orace, "Éléments pour une autostéréotypie », Poétique, n. 125, février 2001, pp. 17-31.

20. Stéphanie Orace, op. cit., p. 19.

21. Ibid.

22. Op. cit., p. 29.

23. Stéphanie Orace, op. cit., p. 21.

24. Op. cit., p. 22.

25. Op. cit., p. 21.

26. Junky, p. 126.

27. Le Camé, pp. 159-160.

28. The Soft Machine, p. 7.

29. The Naked Lunch, p. 182.

30. Le Festin nu, p. 252.

31. The Soft Machine, p. 7.

32. The Soft Machine, p.156. The Naked Lunch, p. 157 et p. 160.

33. Trilogie, p. 140.

34. Philippe Mikriammos, William S. Burroughs, la vie et l'œuvre, Seghers (coll. «Littérature »), Paris : 1975, p. 149. Le texte de cet entretien n'existe qu'en français, dans une traduction de Philippe Mikriammos. 


\section{RÉSUMÉS}

L'auteur américain William Burroughs, dans sa Trilogie des années 1960 à 1968 (The Soft Machine, The Ticket That Exploded, Nova Express) a largement recouru, via sa technique du "cut-up » ou découpage/remontage de textes, à des emprunts littéraux ou indirects à toutes les littératures et paralittératures, et plus globalement à des procédures de reprise voire de ressassement continuel de textes, sous forme littérale ou tronquée. Nous nous proposons d'étudier la manière dont, de livre en livre, William Burroughs construit un véritable réseau de stéréotypes personnels qui lui permettent de mettre en place une identité textuelle forte, selon ce qu'on pourrait qualifier d' autostéréotypie (Stéphanie Orace et Jean-Louis Dufays). L'application de la théorie de l'autostéréotypie sur un texte "résistant" comme le texte cut-up burrougshien permet de dépasser les limites des différents théories de l'intertextualité (transtextualité, autotextualité, intratextualité) et de tenter une théorisation à un niveau plus global des phénomènes de reprises textuelles littérales, tronquées, réécrites ou remontées sur un corpus de textes défini.

The American writer William Burroughs, in his Trilogy written and published from 1960 to 1968 (The Soft Machine, The Ticket That Exploded, Nova Express) has largely used, via his "cut-up" technique of cutting/reassembling different texts, directly or indirectly, every type of texts and literatures, including popular literatures. More generally he used some techniques of repeating a continuous text, literally or incompletely. We study the way in which, book after book, William Burroughs built a network of personal stereotypes which enable him to establish a strong textual identity, according to what is essentially called "autostéréotypie" (Stephanie Orace and JeanLouis Dufays). The application of the theory of "autostéréotypie" on "resisting" texts as the cutup burrougshian text enable to go further than with the use of the numerous theories of intertextuality ("transtextualité", "autotextualité", "intratextualité"); it also enables to theorize to a more global level the use of textual rewriting on a certain kind of text.

\section{INDEX}

Mots-clés : réécriture, intertextualité, stéréotype, autostéréotypie, cut-up, collage, montage

\section{AUTEUR}

\section{BENOÎT DELAUNE}

Docteur en littérature générale et comparée 